\title{
Characterization of the spatial resolution capability of a novel carbon fiber strain sensor based on characteristic impedance measurements
}

\author{
R. Höhne ${ }^{1}$, P. Kostka ${ }^{1}$, N. Modler $^{1}$ \\ ${ }^{1}$ Institute of Lightweight Engineering and Polymer Technology, Faculty of Mechanical Science and \\ Engineering, Technical University of Dresden, Holbeinstraße 3, 01307 Dresden, Germany \\ robin.hoehne@tu-dresden.de
}

\begin{abstract}
:
There is a wide application spectrum of spatial resolving strain sensors for SHM applications in industry in order to provide significant information about the condition of the structure. In this context, a novel carbon fiber strain sensor is developed. The unique feature of the sensor is a fiber-break resistive measurement principle. The proposed strain sensor is based on characteristic impedance measurements of a two-core electrical transmission path, whereby at least one conductor consists of aligned carbon fibers fragments. The electrical time domain reflectometry (ETDR) was used in order to realize a spatial resolved strain measurement. In this contribution, the spatial resolution capability of the sensor was experimentally investigated in a cantilever arm test device. Furthermore, a Finite difference time domain (FDTD) model was established in order to simulate the ETDR signals. Special emphasis was on the identification of the relation in between electrical and mechanical behavior of the sensor. As a result, a spatial resolution in the low single-digits mm-range for engineering relevant strains was experimentally identified. The established FDTD model delivers results that are in good agreement with the experiments and thus constitutes the basis for a more comprehensive numericalassisted characterization of the sensor.
\end{abstract}

Key words: strain measurement, strain gauge, SHM, carbon fibers, electrical time domain reflectometry, finite difference time domain

\section{Introduction}

In many structural health monitoring concepts, strain gauges are commonly used. These strain gauges provide the required data for the prediction of the residual strength and durability of a component, such as an turbine blade or a car body part. In the case of complex shaped and loaded structures, the knowledge of spatial resolved strain fields is essential. Thus, there is a growing need of industry for spatial resolving strain sensors for SHM applications.

In this context, current strain measurement systems like strain gauges or fiber optic sensors have some deficits regarding the complexity (multi-channel installation and processing), cost and design (weight and installation space). The challenge is to make a strain measurement system available, that combines a high spatial resolution with manageable complexity, low weight and low costs.

An innovative sensor technology has switched into the focal point of research at the Institute of
Lightweight Engineering and Technology. The interdisciplinary development of the sensor technology combines the knowledge of material science, production processes of fiber reinforced plastics as well as picosecond impulse technique. The unique feature of the technology is a novel measurement principle based on carbon fibers fragments in combination with the electrical time domain reflectometry (ETDR). The synthesis of interdisciplinary findings makes the sensor technology a promising candidate to overcome current burdens of spatial resolved strain measurement.

\section{Measurement principle and sensor configuration}

The focused sensor patch basically represents a microstrip line, consisting of a brittle carbon fiber (CF) roving and a copper strip which are embedded in a glass-fiber epoxy (GF-EP) carrier material and spaced by a single GF-EP layer, see Figure 1. The measurement principle is based on the reversible opening and closing 
of single filament fragments of the CF-roving. Thus, the proposed strain sensor is based on a fiber-break resistive measurement principle enabling much higher resistance changes compared to conventional carbon fiber sensors using mainly the piezoresistive effect. The measurement principle was phenomenologically described in the preliminary work by means of microscopy images of the CF-roving during tensile tests. Additionally, the integral sensor resistance as a function of the homogeneous strain over the sensor length was determined [1]. Furthermore, the endurance of the sensor was investigated in cyclic tests, showing a stable functionality up to 106 cycles [2].

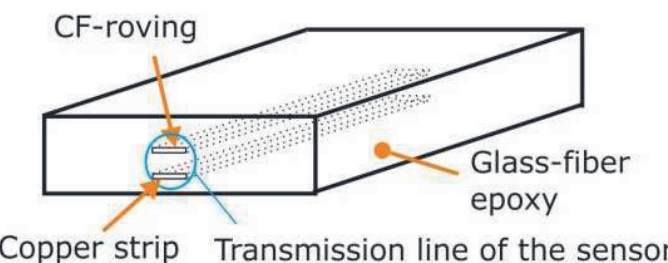

Figure 1: Schematic setup of the carbon fiber strain sensor showing the realization of the microstrip line embedded in a carrier material.

The observed immense resistance changes of the CF-Roving result in impedance changes of the micro strip that is sufficiently high to be measured by means of the ETDR. In [1] the potential of the strain sensor with spatial resolution by means of the ETDR was investigated the first time. The investigations highlight the sensitivity of the sensor to varying strain profiles. However, only homogeneous strain profiles were investigated and no simulations were performed.

\section{Electrical time domain reflectometry}

The ETDR features a fast signal acquisition as well as potentially cheap and easy to miniaturize electronics with low energy consumption enabling handheld diagnostic units. The measurand in the ETDR are reflections of an incident electrical wave at discontinuities of the characteristic impedance along the transmission line. The characteristic impedance based on the telegrapher's equation is

$$
Z=\sqrt{\frac{R^{\prime}+j \omega L^{\prime}}{G^{\prime}+j \omega C^{\prime}}}
$$

where $R$ is resistance, $L$ is inductance, $G$ is conductance and $C$ is capacitance. The apostrophe denotes the length-specific character. In case of a single discontinuity, e. g. from local deformations or damage of the line, the reflection coefficient is

$$
\rho=\frac{E_{i}}{E_{r}}=\frac{Z_{L}-Z_{0}}{Z_{L}+Z_{0}}
$$

where $E_{i}$ and $E_{r}$ are the voltage levels of incoming and reflected signal, respectively, and $Z_{L}$ and $Z_{0}$ are the electrical impedances of two different sections of the transmission line. In a typical application, a single measurement delivers a sampled sequence of up to several thousands of signal values depending on the spatially distributed properties of the transmission path.

In the here investigated case, the focused transmission line exhibits multiple discontinuities, due to the locally opened fiber breaks in the CF-roving conductor. Between the discontinuities re-reflections occur that affect the travelling voltage wave. This effect has to be taken into account, in order to calculate the spatial characteristic impedance correctly. Solving the inverse problem of characteristic impedance calculation and consequently of the strain distribution determination, is a challenging task [3]. Instead, the here presented analysis considers the reflected voltage signal, which is sufficient for an assessment of the sensor's spatial resolution and sensitivity.

\section{Test setup}

A test device was built for the experimental investigation of the spatially resolved strain measurement. A prototypical ETDR sensor patch according the configuration shown in Fig.1, was clamped in a cantilever arm configuration, see Figure 2a. The sensor patch was connected to a ETDR-device (TDR-3000, Sympuls Aachen $\mathrm{GmbH}$ ) using a coax cable. The microstrip line of the sensor was terminated by means of a potentiometer.

The sensor patch was loaded with a point load at the free end. The load level was fixed for each test, by hanging a weight of $100 \mathrm{~g}$ at the free end. The maximum strain on the patch surface at the clamping is calculated as follows:

$\varepsilon=\frac{F l}{E I} e$.(3)

where $F$ is the applied force at the free end, $I$ is the free bending length, $E$ is the Young's modulus, $I$ is the moment of inertia of the patch cross section and $e$ is the distance of the CFroving to the bending neutral axis. In case of a cantilever arm configuration, a linear 
decreasing strain profile occurs at the sensor patch during bending, see Figure $2 \mathrm{~b}$. An overall maximum strain of approximately $0.15 \%$ at the clamping can be realized in the investigated sensor/test device configuration. By shifting the sensor patch in the clamp, the free bending length and thus the strain profile along the sensor patch can be accurately varied. ETDR measurements were performed for several sensor positions in the clamping.

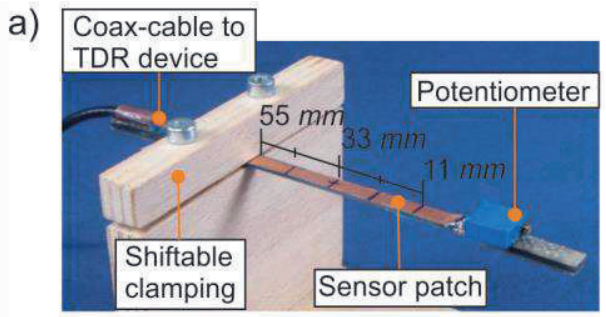

b)

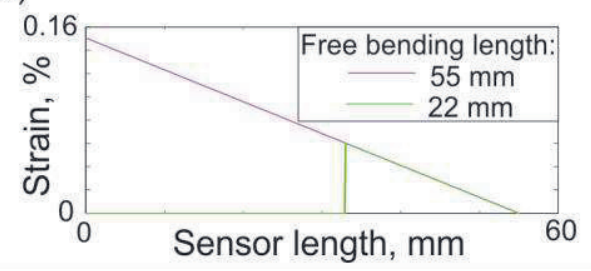

Figure 2: Clamped sensor patch in test device in case of a free bending length of $55 \mathrm{~mm}$ (a) and idealized strain profiles, exemplary shown for $55 \mathrm{~mm}$ and $22 \mathrm{~mm}$ free bending length (b).

\section{Experimental results}

The measured ETDR signal is a function of the free bending length, see Figure 3 . Based on the change with respect to the reference signal, the positon of the clamp can be identified. The measured values show a spatial resolution in the low single-digits $\mathrm{mm}$ range for the investigated sensor configuration. As the ETDR signal is related to the existing strain profile, the signal values decrease at reduced bending length. However, the signal change is significant, though the apparent strains are below $0.15 \%$. It should be noted that the measured signal cannot be directly associated with the strain profile. The identification of the relation between the signal, respectively the characteristic impedance, and the strain is part of ongoing work. Besides the aforementioned re-reflections due to multiple discontinuities also damping effects due to a high resistivity of the transmission line have to be considered.

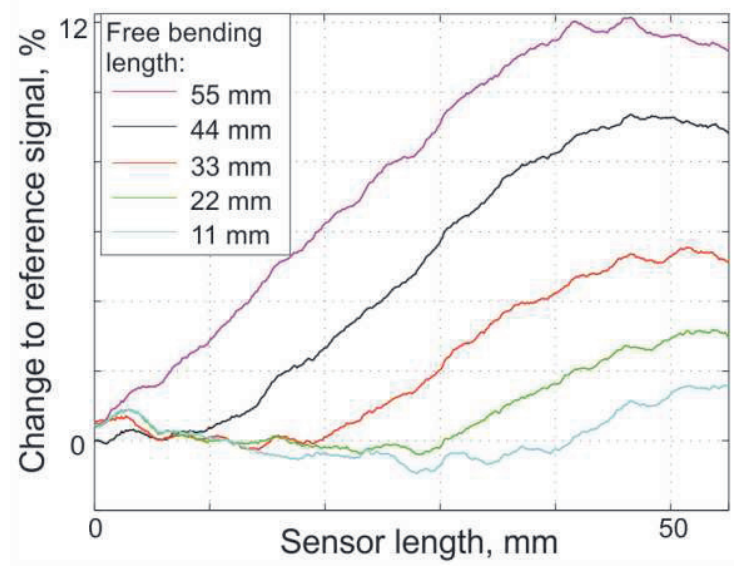

Figure 3: Change of ETDR signal for different bending length of the sensor patch.

\section{FDTD-Simulation model}

A numerical finite-difference time-domain (FDTD) model is developed in order to simulate the behavior of the sensor and to simulatively support the validation of the sensor. The FDTD method is one of the most successful explicit and direct numerical methods to solve large and complex electromagnetic field problems [3]. The method enables the simulation of the electromagnetic field propagation inside the sensing microstrip. Its greatest benefit is the simple and straightforward approach allowing a memory and computationally efficient implementation [3]. The open source software openEMS v0.0.v35, a fully-vectorial threedimensional electromagnetic field solver using the FDTD method, was chosen for the simulation. openEMS provides a user friendly and flexible scripting interface as well as a graphical user interface as a structural 2D/3D viewer [4]. In this investigation, a Matlab based implementation is used.

The developed model is shown in Figure 4. The model is composed of two sections. The sensor section represents the transmission line inside the sensor patch. Here, the transmission line is modelled as a microstrip, where the CF roving is modelled as upper conductor, the copper strip is idealized as ground plane and the glassfibre reinforced layer represents the dieelectric substrate. The upper conductor is grouped in several material sections, in order to allow an assessment of a conductivity profile to the mechanically loaded sensor patch. To each of the sections, a separate conductivity value can be assigned. For the unloaded sensor patch a uniform conductivity over the sensor patch was modelled. The initial material settings are given in Table 1.

The device section is a rough model of the ETDR-device, in which the signal generation 
and monitoring is performed. The transmission line is again modelled as microstrip with copper conductors and a FR4 as dielectric substrate.

In openEMS, the signal excitation and measurement are realized at defined planes. In

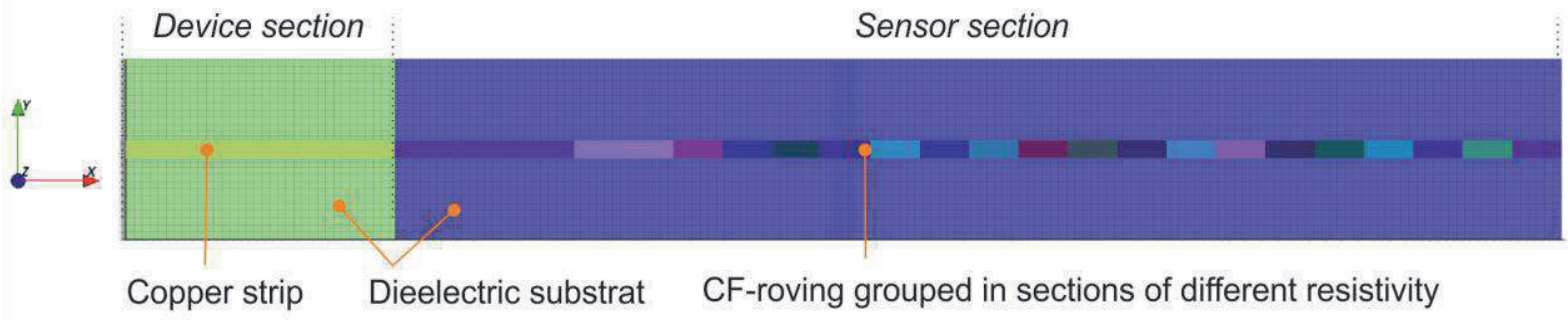

Figure 4: FDTD simulation model developed in openEMS.

Table 1: Material properties assigned to the FDTD model.

\begin{tabular}{ll}
\hline Property & Value \\
\hline $\begin{array}{l}\text { Initial conductivity of CF- } \\
\text { roving }\end{array}$ & $4.45 \cdot 10^{4} \mathrm{~S} / \mathrm{m}$ \\
$\begin{array}{l}\text { Conductivity of copper } \\
\text { Relative permittivity in } \\
\text { sensor section (typical for } \\
\text { glass-fiber layer) }\end{array}$ & $58.0 \cdot 10^{6} \mathrm{~S} / \mathrm{m}$ \\
$\begin{array}{l}\text { Relative permittivity in device } \\
\text { section (typical for FR4 } \\
\text { substrate) }\end{array}$ & 4.66 \\
\hline
\end{tabular}

For the generation of a customized excitation, an excitation function as well as an electric field vector at the excitation plane has to be defined. The correct modelling of a realistic excitation, especially its rise time and voltage amplitude, has an important impact on the modelling accuracy and thus was specifically focused. The gaussian function in time domain was used in order to model the characteristic of the impulse excitation of the used ETDR-device:

$f(t)=a e^{-\frac{(t-b)^{2}}{2 c^{2}}}$

with proper values of the parameters $a, b$, and $c$. For the excitation signal, only the rising edge of the gaussian function was used. The advantage of the gaussian function is the smooth transition at the beginning and end of the impulse. On the one hand, this best fits the realistic impulse signal of the ETDR-device. On the other hand, the bandwith of the impulse signal is not affected by abrupt signal changes. A normalized gaussian function with the same rise time as the ETDR-device was created by manually identifying the parameters:

$a=1 \quad b=1.7 \cdot 10^{-8} \quad c=4.6 \cdot 10^{-9}$ the presented model, two planes for excitation and monitoring (current and voltage) are located in the device section. The location was selected so that the impulse generation is completed before the impulse front enters the sensor section.

Sensor section

The resulting rising edge of the normalized gaussian function is used as excitation, see Figure 5a.

Furthermore, the amplitude of the excitation was set by scaling the e-field at the excitation plane. The e-field is set in such a way, that a voltage impulse of $0.5 \mathrm{~V}$, which is consistent with the real value of the ETDR-device, is generated. The measured signal at the measurement plane for an unloaded sensor patch can be seen in Figure $5 \mathrm{~b}$. In this case, the measured signal shows the generated impulse at the beginning. Subsequently, reflections occurring along the transmission line, superimpose the maintained constant voltage level.
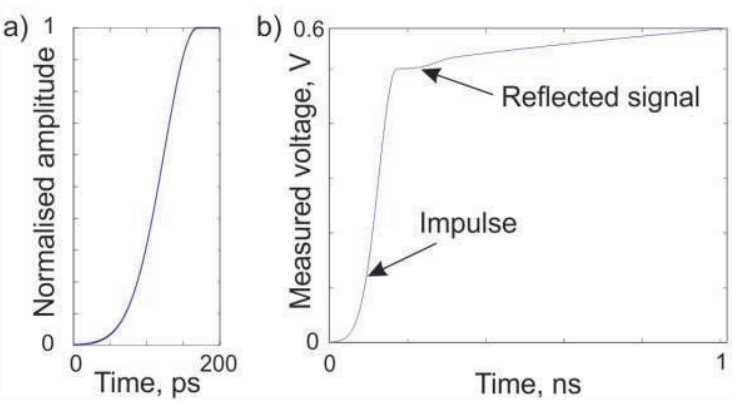

Figure 5: Generated rising edge of gaussian function using the identified real constants (a) and measured voltage for the unloaded sensor patch (b).

\section{Modeling of ETDR signal due to mechanical bending}

In order to simulate the ETDR signal of the sensor patch due to bending, a relation between the electrical and mechanical behavior of the sensor patch is of interest. In case of the FDTD model, the conductivity profile - the variance of the conductivity along the sensor patch - needs to be identified.

Therefore, the relationship between strain and resistance of the sensor patch, using the 
tension test device described in [1], was identified, see Figure 6a. Based on the measured resistance $R$ the conductivity $\mathrm{K}$ is calculated as

$\kappa=\frac{l}{A R}$

where $A$ is the cross-section and $/$ is the length of the conductor (CF-roving). This equation is used for the sake of simplicity, though it is only valid for homogeneous cross-section along the conductor. A value of $0.4 \mathrm{~mm}^{2}$ for the crosssection of the roving based on microscopy images was used.

In order to identify the conductivity profile, beside the identified strain-conductivity relation, also the strain profile was calculated according to Eq. 3. Based on the strain-conductivity relation and the strain profiles, the conductivity profiles were calculated for each of the prior conducted bending tests, see Figure $6 \mathrm{~b}$. A discretized profile was assigned to the defined material section of the CF-roving in the FDTD model. The calculation of the resulting ETDR signal was performed subsequently.

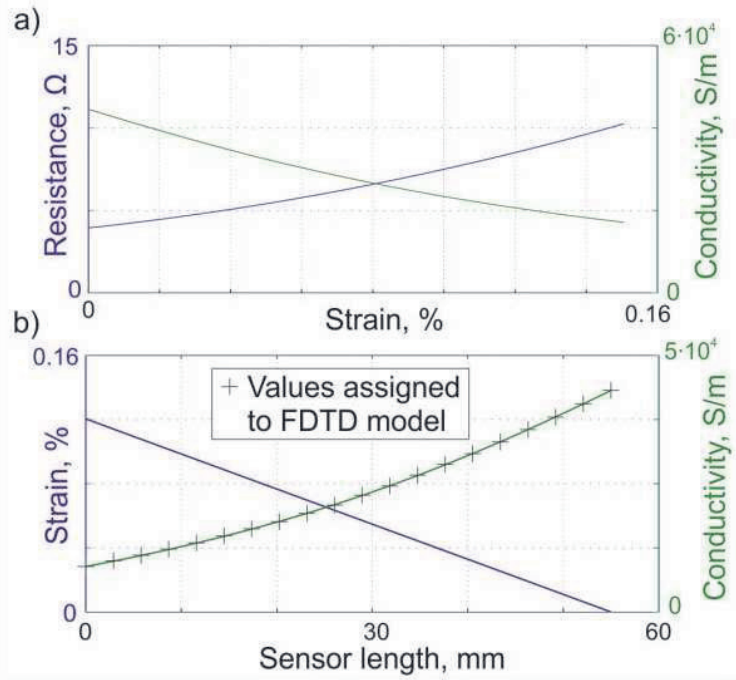

Figure 6: Experimentally identified relation between electrical and mechanical behavior of the sensor patch (a) and calculated strain and conductivity profiles along the sensor patch for free bending length of $55 \mathrm{~mm}$ (b).

\section{Comparison of experimental and numerical results}

The change of the simulated reflected signals with respect to the reference signal are shown in Figure 7. The simulation results are in good agreement with the experimental data (see Figure 3). Qualitative accordance for measured and simulated signals can be found for the smooth transitions at the beginning and end as well as for the decreasing signal value with reduced free bending length.

The quantitative deviations can be explained by the simplifications made for the modeling. This includes the idealization of the sensor setup as microstrip, the simplified calculation of the conductivity as well as the discretization of the conductivity properties along the sensor.

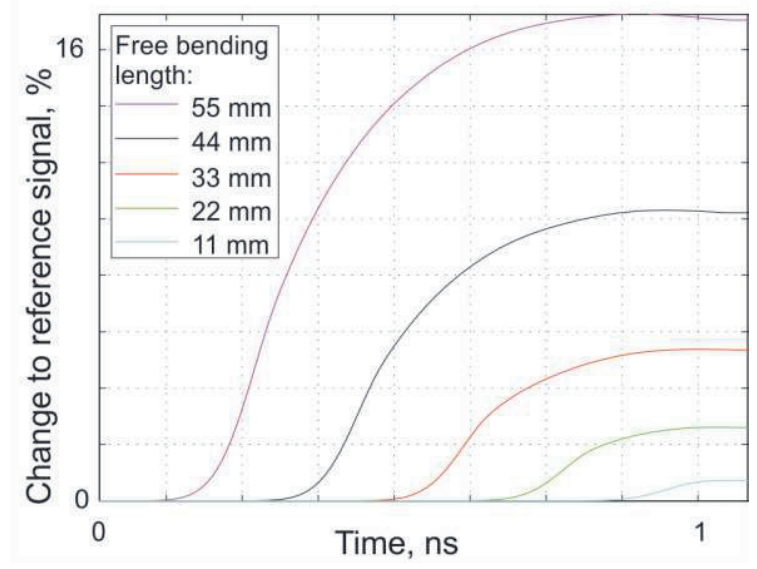

Figure 7: Change of simulated ETDR signal for different bending length of the sensor patch.

\section{Summary}

The investigation highlights the immense potential of the presented carbon fiber strain sensor for a spatial resolved strain measurement.

A bending test device, which enables the variation of the free bending length of the sensor patch, was used for the experimental characterization. ETDR signals were measured for five different bending lengths (55 $\mathrm{mm}$ to 11 $\mathrm{mm}$ ). A maximum strain of approximately $0.15 \%$ was realized next to the clamping. The experimental results show a spatial resolution capability in the low single-digits $\mathrm{mm}$ range.

A FDTD model was established in order to simulatively support the experimental work. A prerequisite in order to model the ETDR signal due to mechanical bending was a relation that describes the electromechanical behavior of the sensor. This was identified by means of tensile tests of the sensor patch with simultaneous measurement of resistance as a function of the homogeneous strain. It was shown that the identified relation can be used in order to simulate the behavior of the sensor at more complex strain profiles. Therefore, the conductivity was calculated based on the measured resistance and the geometry of the sensor. The numerical and experimental results show a qualitative accordance regarding the signal characteristic. 
The established FDTD model constitutes the basis for a numerical-assisted characterization of the sensor properties. Furthermore, the model is a powerful tool in order to develop an appropriate algorithm for a spatial resolved characteristic impedance calculation. A further development of the presented FDTD model will be followed up by a comprehensive numerical study in future work.

\section{Acknowledgement}

The 3 Ccar project has received funding from ECSEL Joint Undertaking under grand agreement No. 662192. This Joint Undertaking received support from the European Union's Horizon 2020 research and innovation programme and Germany, Austria, Czech Republic, Romania, Belgium, United Kingdom, France, Netherlands, Latvia, Finland, Spain, Italy, Lithuania.

\section{References}

[1] R. Höhne, T. Ehrig, P. Kostka, N. Modler, Special Issue of Materials Science and Engineering Technology, accepted for publication. 10.1002/mawe.201600628

[2] R. Höhne, P. Kostka, N. Modler, 17th European Conference on Composite Materials, Munich, Germany, 26-30th June 2016. Proceedings available as OpenAccess half a year after conference

[3] A.Scheuermann et al: Spatial Time Domain Reflectometry (spatial TDR) in geo-environmental engineering. Sensors Applications Symposium (SAS), 2014 IEEE.

[4] T. Liebig, A. Rennings, S. Held, and D. Erni: openEMS - A free and open source equivalentcircuit (EC) FDTD simulation platform supporting cylindrical coordinates suitable for the analysis of traveling wave MRI applications. Int.J.Numer.Model, 2013, 26, 680-696.

[5] http://openems.de; last accessed: 03/2017 